\title{
Challenges and Best Practices in Perspective
}

In this chapter, we compare the challenges and best practices identified in the country chapters so that the in-depth analysis of selected Member States is complemented with a broader overview. In doing so, we aim to provide a better understanding of the practical implementation of the new European Union (EU) pharmacovigilance legislation across Member States.

The chapter is structured as follows. First, the national pharmacovigilance systems are compared regarding their structural factors as well as their institutional frameworks. The second section deals with the main finding of this study, namely the underreporting of adverse drug reactions (ADRs) in the Member States. This finding is substantiated by identifying problems in terms of batch numbers for biological medicinal products (biologicals), scientific evaluation, signal detection and information processing. Finally, we present a comparison of factors contributing to underreporting. The key factors in this respect are as follows:

- Lack of awareness

- Complexity of ADR reporting

- Lack of cooperation

- Interconnectivity problems

Key factors and the underlying reasons have been discovered through repeated rounds of trial and error, relying on pharmacovigilance literature, reports on the effectiveness of pharmacovigilance systems and interview data gathered for the selected cases. By probing the empirical evidence with a variety of categorisations, we aimed to find the right balance in the accurate reporting of specific reasons while relying on key factors for meaningful comparative analysis and generalisation. 


\subsection{Pharmacovigilance Systems}

The objective of this section is to provide a comparative overview of the national pharmacovigilance systems. As indicated in Chapter 3, EU pharmacovigilance is a multi-level system where actors are linked through multiple inter-institutional relations. Adding to this complexity, national pharmacovigilance systems themselves comprise a multitude of actors, which cooperate in line with patterns of implementation (see Chapter 5).

\section{Level of Centralisation}

Due to different healthcare policies and institutions, there is considerable variation in the level of centralisation. Whereas some Member States have centralised systems of pharmacovigilance, others have decentralised systems. Germany, the United Kingdom, Finland and Poland have centralised systems; Portugal and France have decentralised systems. The decentralised systems are characterised by having regional centres for collecting and processing pharmacovigilance data.

The level of centralisation, however, is not set in stone. Whereas Portugal has moved from a centralised system to a decentralised system in the early 2000s, the United Kingdom has set up regional Yellow Card centres.

Although the system in Germany is centralised, sectoral associations of physicians and pharmacists provide functional links between patients, healthcare providers and national competent authorities. Hence, the system can be characterised as highly complex. Depending on the type of medicine, two different national agencies are at the centre of ADR reporting.

\section{Supervision}

In terms of supervision, the national agencies implementing pharmacovigilance are usually supervised by the health and social security ministries. In implementing pharmacovigilance, the agencies are also accountable to respective ministries. In the sample, however, Finland and Poland are exceptions. Even though Fimea and URPL are officially headed by ministries that set the legal guidelines, they are $d e$ facto independent and do not have to justify their decisions.

\section{Separate Systems for Biologicals}

There is considerable variation among Member States regarding the classification of biologicals. Whereas some Member States operate separate systems for biologicals, others treat biologicals as part of their general pharmacovigilance systems. In the sample, only Germany operates a separate system for biologicals. In line with its 
complex system, there are two national agencies operating in Germany, one dealing with synthetic medicines and the other one with biologicals. Finland and Poland have a system for biological vaccines. However, Portugal, France and the United Kingdom have no separate system. ${ }^{7}$

This variation is not surprising, given the fact that there is no unambiguous agreement among Member States about how to classify biologicals (see Klein et al. 2015).

\section{Legal Requirements}

In the sample, almost all Member States have legal requirements for healthcare professionals to report ADRs, i.e. Portugal, France, Finland and Poland. In France, this includes brand names and batch numbers of biologicals (Vermeer et al. 2015: 8). In Germany and the United Kingdom, there are no legal requirements in place, and hence ADR reporting in these countries is based on professional obligations and codes of conduct. In Poland, there are legal requirements but no punishment in the case of non-compliance.

Patients are generally not required to report ADRs, in contrast to marketing authorisation holders that are legally obligated to report them in all countries under investigation.

\section{Prescribing Medicines}

There is also considerable variation among Member States regarding the practice of prescribing medicines. In line with the different national health systems, doctors and pharmacists might substitute different medicines with the same active substance. Previous studies have shown that prescribers often communicate only the international non-proprietary name (INN) in ADR reporting (Dolinar and Reilly 2014). Merely indicating the INN in an ADR report can be misleading, though, as two different medicines (the original biological and the new biosimilar) may have the same non-proprietary scientific name. However, while some stakeholders call for a change to such practices, the majority of Member States maintain that biosimilars and reference products should be closely aligned and that using different INNs undermines such an alignment (see European Commission Pharmaceutical Committee 2013).

7 Despite the absence of a separate system, biologicals receive particular attention in the United Kingdom. Biologicals are discussed by MHRA experts in separate meetings. 


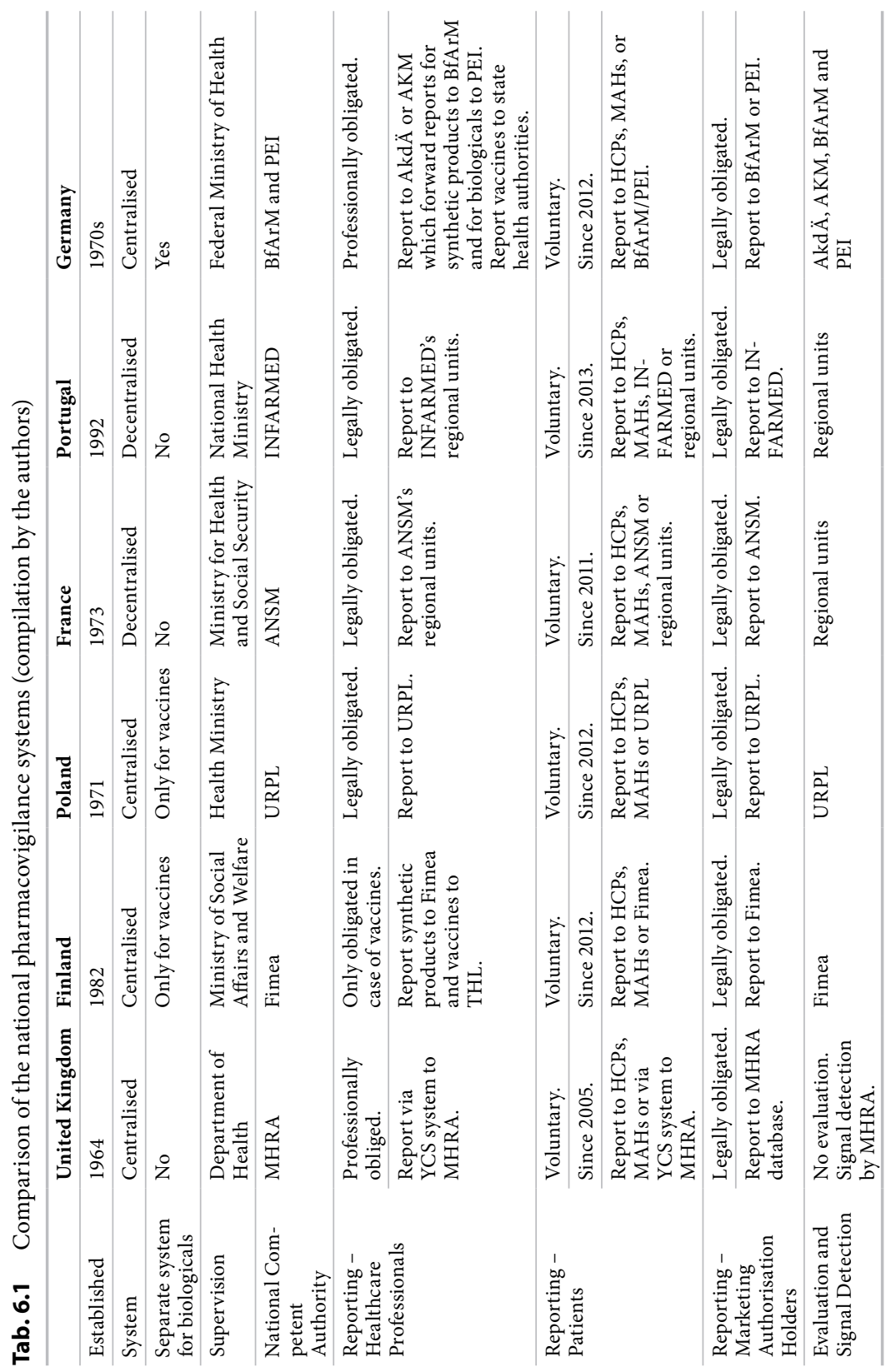




\subsection{Major Challenges}

The objective of this section is to present underreporting as the main finding of this study.

\section{Underreporting}

The most important challenge for the effective implementation of Directive 2010/84/ EU is the underreporting of ADRs. In all Member States in the sample, respondents highlight this (the United Kingdom, Poland, France, Portugal and Germany). This study thereby confirms previous studies based on the comparative analysis of cases selected on their variation in terms of national health systems.

This is remarkable, given that in 22 countries (79 percent) reporting of ADRs is a legal obligation for healthcare professionals, and the percentage of countries with mandatory reporting of ADRs for vaccines is even higher, i.e. 26 countries (89 percent) (Šarinić et al. 2016).

The highest percentage (38 percent) of all ADR reports is received via webbased applications, even though the number of Member States having web-based reporting is lower than the number of Member States with mail-reporting channels available (21 vs. 28 , respectively) (Šarinić et al. 2016). In our sample, only a minority of the French regional pharmacovigilance centres appeared to have websites and thereby web-based reporting formats. In Finland, even though online reporting is possible for physicians and pharmacists, patients have to resort to regular mail. All Member States have ADR reporting forms available on their national competent authorities' websites (SCOPE).

There are two important aspects to underreporting: the quantity and quality of information. Quantity refers to the number of ADR reports; quality refers to the value of information. Both dimensions are sometimes mutually exclusive, as increases in quantity might lead to decreases in quality. Whereas quantity of information is important, an appropriate level of quality is essential for effective pharmacovigilance. Some respondents fret about incomplete reports, requiring follow-up and thereby further increasing the workload (Germany). Other respondents were rather satisfied with the quality of ADR reports but criticized the number of reports submitted (Poland).

\section{Information Overload}

Furthermore, information overload can make it difficult to detect signals (Germany). This problem is exacerbated by the fact that the legislation allows the collection of information from all possible and available sources such as patients and literature 
reports. This also leads to an increased pool of information from which relevant signals can be analysed (Borg et al. 2015: 121).

There is considerable variation in the Member States regarding evaluation and signal detection.

In the United Kingdom, there is no evaluation of reports before signal detection; due to the large quantity of information, signal detection has been automated. After prioritising detected signals, the national competent authority, the Medicines and Healthcare Products Regulatory Agency (MHRA), forwards serious cases to EMA.

\section{Causality Assessments}

In the two decentralised systems, Portugal and France, ADR reports are collected and evaluated by the regional units. However, problems in terms of quantity and quality might lead to problems in terms of causality assessments (France). After the evaluation, including the causality assessment, the reports are forwarded to the national competent authorities. Reports by marketing authorisation holders are forwarded directly to the national agencies.

\section{Traceability of Biologicals}

Previous studies showed that the reporting of batch numbers and traceability of biologicals is subject to considerable variation among Member States (Vermeer et al. 2015; see also European Commission 2015). Various studies on ADR reporting revealed that batch numbers were available for only a limited number of suspected biologicals (Vermeer et al. 2013: 620-621; see also Vermeer et al. 2015: 6).

Again, this study substantiates this. Respondents in various Member States in the sample confirm that reporting batch numbers remains a challenge (the United Kingdom). Batch numbers are reported infrequently (France) or not at all (Poland). Only in Finland did the reporting of batch numbers satisfy the respondents which has been corroborated by Fimea's statistics.

\section{Strengthening Patient Involvement}

One of the aims of Directive 2010/84/EU is to strengthen patient involvement in the safety monitoring of medicines. All 28 Member States have patient reporting systems in place, with the majority initiating them in 2012-2013 (although the first Member States to introduce this process did so only starting in 1968 and the second time in 1996). Overall, the number of individual patient reports from the European Economic Area has increased over the two and a half years of the reporting period by around 50 percent. This includes ADR reports not submitted 
by other reporters such as healthcare professionals, which represent information that would not otherwise be captured (Commission 2016).

However, reporting ADRs regarding biologicals and respective traceability remains a challenge when it comes to patient reporting. For instance, batch numbers have to be displayed on each package in the United Kingdom, but patients often do not have access to the package when the drug is administered in a hospital setting.

\subsection{Comparison of Factors Contributing to Underreporting}

The objective of this section is to compare factors contributing to underreporting and thereby to put into comparative perspective the challenges and best practices in national pharmacovigilance systems.

\subsubsection{Lack of Awareness}

As described above, awareness as an analytical category for comparison includes a number of factors, such as not only the general awareness of the obligation to report, but also indifference regarding the importance of ADR reporting.

Member States have focused on informing healthcare professionals and patients about the importance of ADR reporting, particularly regarding biologicals (see Vermeer et al. 2015). The importance of raising awareness has been emphasized as part of the SCOPE implementation project (Jadeja and Barrow 2016: 59).

However, the study reveals that lack of awareness is a prevalent feature in all the Member States of our sample (the United Kingdom, Finland, Poland, France, Portugal and Germany). Yet lacking awareness concerns healthcare professionals and patients in varying degrees. Whereas in Finland, most healthcare professionals are aware of their obligation to report ADRs, the level of public awareness is particularly low. Similarly, in the United Kingdom about 80 percent of healthcare professionals know about the Yellow Card Scheme, while only 10 percent of patients had heard about this.

\section{Lack of Awareness Regarding Biologicals}

In some Member States, lack of awareness regarding biologicals seems to be particularly severe (Poland, Portugal). This is an interesting finding because Portugal's decentralised system seems well-equipped regarding a key recommendation about 
the role of regional pharmacovigilance centres for awareness-raising campaigns (Jadeja and Barrow 2016: 59-60). Also, Poland revealed that problems regarding ADR reporting of biologicals mainly stemmed from its inexperience and thus its unawareness in the field.

\section{Legal Framework}

As explained in the fundamentals regarding pharmacovigilance (see Chapter 2), legal ramifications of ADR reporting due to liability claims is a specific factor of awareness. While healthcare professionals are bound by the legal systems in which they operate, awareness regarding the legal ramifications and how they affect ADR reporting is a key recommendation (see Chapter 7).

In general, the possibility of liability claims presents an important impediment for ADR reporting. The SCOPE data demonstrates that the quantity can be enhanced by exempting healthcare professionals from liability when they report ADRs that possibly resulted from medication errors (Šarinić et al. 2016: 218). In this context, our study reveals that liability claims are feared by healthcare professionals in Poland, France and Portugal, which impedes ADR reporting in these countries.

In a similar vein, ADRs and the reporting thereof are sometimes perceived as a failure of healthcare professionals associated with a threat to professional reputation.

\subsubsection{Complexity of ADR Reporting}

Complexity as an analytical category encompasses a number of factors related to the task of ADR reporting, including not only reporting logistics, but also constraints in the work environment of healthcare professionals.

In our study, factors impeding ADR reporting due to complexity have been frequently highlighted by respondents and it appears that such complexity affects both the quantity and quality of ADR reporting.

For instance, in Finland only healthcare professionals (physicians and pharmacists) are able to report online, while patients and nurses have to report through regular mail. In Germany, duplications are difficult to filter out because strict data protection laws make it difficult to connect databases.

SCOPE data reveals that offering background and supplementary information within the electronic or the paper version of the reporting form enhances the quality of the submitted reports (Jan and Radecka 2015: 63-64). However, a number of respondents, especially in Poland, France and Portugal, cite high workload as an impediment to more effective pharmacovigilance. 
ADR reporting is also perceived as time-consuming and complex (France, Portugal). The necessity of having to be available for follow-up questions further disincentives healthcare professionals, given that reporting is not a one-off activity, but may turn into a lengthy process. This also has negative consequences for data quantity.

\section{Training in Pharmacovigilance}

The lack of quality (and also underreporting and the lack of quantity) are related to the neglect of pharmacovigilance training during medical and pharmaceutical education. This is the case in the United Kingdom and Germany. Finland, however, offers a very elaborate educational system for healthcare professionals regarding pharmacovigilance, and France is one of the first Member States to introduce a master's programme in pharmacovigilance.

The SCOPE data shows that only a minority of the national competent authorities (e.g. the MHRA) offer e-learning tools and online educational materials for healthcare professionals, although these tools are very efficient and considerably improve professional training (Jadeja and Barrow 2016: 57). Yet in order to implement legal provisions of EU pharmacovigilance to full effect, training is essential for internalising practices that are conducive to the realisation of these provisions. Because most ADRs are well-known effects of old drugs, harm might be avoided if healthcare professionals (and also patients) were better trained or at least informed (Moore and Begaud 2010).

In general, national pharmacovigilance systems must be seen as dependent on general policy developments. In the current political climate, one such development is the structural scarcity of funding for regulatory activity.

\section{Financial Resources}

Pharmacovigilance is no exception here, with several Member States in the sample having to fulfil their functions with limited financial resources (e.g. France, Germany). While scarce finances are an issue across the board, the problem is acute in Southern European Member States such as Portugal that were particularly affected by the economic crisis.

\subsubsection{Lack of Cooperation}

Cooperation is an essential analytical category for contextualising individual ADR reporting by healthcare professionals in complex national systems of pharmacovigilance. 
A multitude of actors in national pharmacovigilance systems require cooperation among pharmacovigilance-related institutions in order to ensure system effectiveness. The SCOPE authors recommend fostering the exchange of information and the sharing of best practices among the Member States and the relevant stakeholders (Jadeja and Barrow 2016: 58). Aside from interconnecting state authorities, the SCOPE authors also suggest enhancing collaboration with patient organisations and professional associations on a national as well as an international level (Jadeja and Barrow 2016: 58).

However, there is considerable variation in the Member States due to their institutional differences. At one end of the spectrum is Portugal with the successful cooperation of agencies, healthcare professionals and universities. At the other end of the spectrum is Poland with no cooperation between relevant actors.

\subsubsection{Interconnectivity Problems}

Due to the increase of ADR reporting since the adoption of Directive 2010/84/EU, the technical infrastructure for data processing and interconnectivity of databanks is a particular challenge. In order to cope with increasing data, Member States have introduced new functionalities to reporting systems and cooperation between hospital and pharmacy IT systems (Vermeer et al. 2015: 8).

SCOPE data suggests that sound and uniform IT systems for reporting ADRs on a national as well as an international level would lead to increased efficiency, better data quality and error prevention (Šarinić et al. 2016: 220).

However, the case studies highlight specific problems in terms of interconnectivity. Our study shows that different IT systems and separated online portals are seen as impeding interconnectivity in a number of Member States (the United Kingdom, France and Germany).

In conclusion, it is important to emphasize that healthcare policies of Member States differ considerably. Accordingly, a comparison of challenges and best practices can inform mutual learning, yet such learning is contingent on deep-seated structural and cultural factors that affect the national implementation of EU pharmacovigilance legislation.

Furthermore, broader policy developments have significant effects on the implementation of pharmacovigilance. For instance, due to strict data protection laws, Germany can be characterised as a laggard in pharmacovigilance research (see Douros et al. 2016). Due to the Mediator scandal, France has to be mentioned as well, given that the national pharmacovigilance system is still impeded by systematic difficulties. 
In the final chapter, we suggest several recommendations aiming to cope with these persistent challenges in national ADR reporting systems and thereby to improve the practical implementation of Directive 2010/84/EU.

\section{References}

Borg et al. (2015). European Union Pharmacovigilance Capabilities: Potential for the New Legislation. Ther Adv Drug Safety 6 (4): 120-140.

Dolinar, R., Reilly, M. (2014). Biosimilars Naming, Label Transparency and Authority of Choice - Survey Findings among European Physicians. Generics and Biosimilars Initiative 3 (2): 58-62.

Douros, A. et al. (2016). Pharmakovigilanz in Deutschland. Internist 57: 616-623.

European Commission Pharmaceutical Committee (2015). Overview of Member States Biennial Reports on Audits of their Pharmacovigilance Systems (2013 Reporting Year). Pharmaceutical Committee, 21 October 2015, PHARM 693.

European Commission (2016). Pharmacovigilance Related Activities of Member States and the European Medicines Agency Concerning Medicinal Products for Human Use (20122014), $\operatorname{COM}(2016) 498$ final, Brussels, 08.08.2016.

Jadeja, M., Barrow, P. (2016). Topic 4.3: Awareness Levels. SCOPE Work Package 4 Survey Report.

Jan, T., Radecka, (2015). Topic 4 Review of Reporting Forms. SCOPE Work Package 4 Survey Report.

Klein, K., De Bruin, M. L., Broekmans, A. W., Stolk, P. (2015). Classification of Recombinant Biologics in the EU: Divergence between National Pharmacovigilance Centres. BioDrugs 29: 373-379.

Moore, N., Bégaud, B. (2010). Improving Pharmacovigilance in Europe. The BMJ 340: c1694.

Šarinić, V. M., Di Giusti, M. D., Banovac, M., Skurce, N. M., Gvozdanović, K., Krnic, D., Andrić, A., Šipić, I., Cajko, N., Sudić, D., Lovretić, N. (2016). Topic 1 Audit of National Reporting Systems, Topic 1a Medication Errors, Topic 2 Patient Reporting, Topic 5 Review of IT Systems and Special Form of Reports. SCOPE Work Package 4 Survey Report.

Vermeer, N. S., Straus, S. M. J. M., Mantel-Teeuwisse, A. K., Domergue, F., Egberts, T. C. G., Leufkens, H. G. M., De Bruin, M. L. (2013). Traceability of Biopharmaceuticals in Spontaneous Reporting Systems: A Cross-Sectional Study in the FDA Adverse Event Reporting System (FAERS) and EudraVigilance Databases. Drug Safety 36: 617-625.

Vermeer, N. S., Spierings, I., Mantel-Teeuwisse, A. K., Straus, S. M. J. M., Giezen, T. J., Leufkens, H. G. M., Egberts, T. C. G., De Bruin, M. L. (2015). Traceability of Biologicals: Present Challenges in Pharmacovigilance. Expert Opinion on Drug Safety, 14 (1). 
Open Access This chapter is licensed under the terms of the Creative Commons Attribution 4.0 International License (http://creativecommons.org/licenses/by/4.0/), which permits use, sharing, adaptation, distribution and reproduction in any medium or format, as long as you give appropriate credit to the original author(s) and the source, provide a link to the Creative Commons license and indicate if changes were made.

The images or other third party material in this chapter are included in the chapter's Creative Commons license, unless indicated otherwise in a credit line to the material. If material is not included in the chapter's Creative Commons license and your intended use is not permitted by statutory regulation or exceeds the permitted use, you will need to obtain permission directly from the copyright holder. 